factores de susceptibilidad genética como la presencia del alelo e4.

En algunos estudios se propone que el alcohol puede tener algún efecto protector contra el deterioro cognoscitivo. Sobre la base del estudio descrito, la conclusión es que el consumo moderado de alcohol es solamente un indicador de un estilo de vida favorable, que es de por sí protector. En cuanto al efecto del alelo e4, es posible que los portadores tengan medios de reparación neural menos eficientes que los no portadores y son, por lo tanto, susceptibles frente a los daños que suele producir el alcohol. (Anttila T, et al. Alcohol drinking in middle age and subsequent risk of mild cognitive impairment and dementia in old age: a prospective population based study. BMJ. 2004 Sept 4(7465); 329:539 E pub 2004 Aug 10, PMID:15304383.)

\section{La atención prenatal y el bajo peso al nacer}

La atención prenatal adecuada suele ser una meta importante en cualquier programa de salud de la mujer, ya que se considera la forma más rentable de mejorar los resultados del embarazo. En el Brasil, la reforma sanitaria de los años ochenta garantizó el acceso universal gratis a todos los servicios públicos bajo el nuevo Sistema Único de Salud. En 1989 se inició un programa destinado a reducir las desigualdades de acceso a la atención de salud en las mujeres, especialmente durante la gestación. El Ministerio de Salud recomendó que toda embarazada comenzara sus visitas a un centro de salud a partir del primer trimestre de la gestación y fuera revisada por un obstetra por lo menos seis veces durante los meses siguientes. Con esta estrategia se procuraba atraer a los servicios de salud a las mujeres de escasos recursos, dada la estrecha relación entre las barreras socioeconómicas y el acceso a una atención prenatal satisfactoria. Sin embargo, se han llevado a cabo muy pocos estudios para evaluar el éxito de esas medidas.

En países en desarrollo, donde las poblaciones podrían beneficiarse más del cuidado apropiado, escasea la información sobre los efectos de la atención prenatal en el bajo peso al nacer (BPN). Es interesante que en otros sitios, principalmente en países industrializados, diversos estudios indican que el aumento de la atención prenatal no ha logrado las reducciones esperadas en el BPN. Con esta perspectiva, un grupo de investigadores brasileños se dedicó a estudiar la situación. Para sus fines, un ensayo aleatorizado controlado hubiera sido ideal, pero poco ético, ya que obligaría a establecer a un grupo no atendido. En este contexto, hay que depender de estudios observacionales, a pesar de que plantean muchos problemas metodo- lógicos de sesgo y factores de confusión. Comparar el uso adecuado con el inadecuado de los servicios tiende a subestimar las ventajas de la atención prenatal, sesgo que se trató de subsanar sopesando la asociación entre esa atención y el BPN. El efecto del uso adecuado de la atención prenatal en el peso del neonato se comparó con el efecto del uso inadecuado y de la total falta de atención prenatal. Los datos de estudio se tomaron de encuestas sobre dos cohortes de nacimiento en Riberão Preto, al sureste del país: una realizada de 1978 a 1979 con 6750 pares de madres y niños, antes del inicio del programa mencionado, y otra de 1994 con 2846 pares, cinco años después de haberse puesto en marcha la estrategia. Las variables maternas independientes fueron edad, escolaridad, situación marital, número de partos, tipo de seguro de enfermedad y hábito de fumar.

Mientras que el uso de la atención prenatal mejoró en el período estudiado, de 39,4\% en 19781979 a $64,0 \%$ en 1994, el BPN aumentó de 7,2\% a $10,7 \%$. En ambas encuestas, las mujeres que no aprovecharon los servicios de salud tendieron a ser menores de 20 años, fumadoras, con menos de 12 años de escolaridad, al menos cuatro embarazos y seguro público. Las madres primíparas tendieron a usar mejor la oportunidad de atención. Solo las de 35 años o mayores de la primera cohorte acudieron al control prenatal menos de las veces recomendadas. Las desigualdades en la atención prenatal aumentaron de 1979 a 1994; mientras menos escolaridad, menos asistencia a los servicios de salud. En ambos grupos, las gestantes que no asistieron a sus citas prenatales mostraron un riesgo significativamente mayor de tener un hijo de peso bajo. En el grupo de 1994, si bien se mantuvo ese riesgo en las que no recibieron ninguna atención prenatal, desapareció la asociación entre el uso inadecuado de la atención prenatal y el BPN. Esto hace pensar que otros factores, ajenos al uso de la atención prenatal, han incrementado el BPN en los hijos de mujeres pudientes y bien cuidadas. La mayor difusión de estadísticas demográficas, la elección más frecuente de intervenciones obstétricas y las nuevas técnicas para conseguir el embarazo parecen asociarse a un aumento en el parto prematuro y BPN, especialmente en mujeres de clase adinerada. La atención prenatal intensa que supera el número de visitas recomendadas según la edad gestacional también se asocia a un mayor número de intervenciones y BPN. En algunos casos, el aumento de partos prematuros refleja los adelantos en medicina perinatal, pues la reducción de muertes fetales en mujeres que han recibido buena atención prenatal también reduce la asociación entre el uso de esa atención y el BPN. Además, el parto por cesárea es más común entre mujeres privilegiadas. Pueden alegarse otras 
posibles explicaciones: errores de medición al calcular la edad gestacional y el número de visitas prenatales, y el hecho de que las posibles ventajas de la atención prenatal son menos pronunciadas a medida que mejora la salud reproductiva de una población. No obstante, los encargados de las políticas sanitarias deben seguir ocupándose de intensificar el cuidado prenatal de las muchas mujeres menos privilegiadas. (Goldani MZ, et al. Trends in prenatal care use and low birthweight in Southeast Brazil. Am J Public Health. 2004;94(8):1366-1371.)

\section{Régimen para reducir la tensión arterial}

Un nuevo informe reitera que la dieta Dietary Approaches to Stop Hypertension o conocida por su sigla DASH, puede llevar a una reducción notable de la tensión arterial. El régimen se basa en frutas, hortalizas, productos lácteos con grasa reducida, granos integrales, aves, pescado y nueces, y reducción de la ingesta de sodio. Este régimen se ha probado en dos ensayos en los que se comparó con la típica dieta estadounidense. En el primer estudio, la ingesta de sal se mantuvo a un promedio constante de $135 \mathrm{mmol}$ al día, es decir un poco menos que la ingesta promedio en los Estados Unidos. El segundo estudio fue un ensayo de eficacia ideado para evaluar los efectos principales e interactivos del régimen DASH y una ingesta reducida de sodio en la tensión arterial. Incluyó a 412 participantes con presión sistólica de 120 a 159 mmHg y diastólica de 80 a $95 \mathrm{mmHg}$. Después de dos primeras semanas consecutivas durante las cuales los participantes se alimentaron con una dieta de referencia de contenido sódico alto, se asignaron aleatoriamente a dos grupos: uno alimentado con el régimen DASH y el otro con una típica dieta estadounidense. Según el diseño cruzado, cada grupo fue expuesto consecutivamente a tres diferentes ingestas de sodio de 30 días cada una, en orden aleatorio. Las tres concentraciones de sodio (alta, intermedia y baja) tenían como objetivos 150, 100 o $50 \mathrm{mmol}$ al día (en la dieta de $2100 \mathrm{kcal}$ ). El contenido de sodio más elevado, típico del consumo estadounidense, era 50\% más alto que el intermedio, mientras que este representaba el límite superior de lo que se recomienda actualmente en el país.

La tensión arterial se midió tres veces antes de la inscripción, dos veces durante el período inicial de dos semanas y dos veces al día durante cinco de los últimos nueve días de cada período de alimentación de 30 días. Cada día se calculaba el promedio de las dos mediciones. Se consideró tensión arterial de inicio el promedio de los cinco pares de valores medidos antes de empezar las dietas asignadas. Los valores finales se obtuvieron del promedio de los cinco pares de mediciones al final de cada período de alimentación de 30 días. Se denominó hipertensión una presión sistólica $\geq 140 \mathrm{mmHg}$ o una diastólica $\geq 90 \mathrm{mmHg}$.

Tanto la reducción de sodio como la dieta DASH produjeron disminuciones de tensión arterial estadísticamente significativas y de importancia clínica en subgrupos definidos inicialmente por su estado de hipertensión, raza u origen étnico, sexo y edad. El extenso análisis de esos ensayos, recientemente publicado, presenta los resultados en la tensión arterial de subgrupos considerados a priori de importancia clínica y de subgrupos mixtos, como edad y estado de hipertensión, raza u origen étnico y el estado de hipertensión y género y raza u origen étnico. Mientras más reducida fue la ingesta de sodio, más se redujo la tensión arterial. Este resultado se observó en todos los participantes, independientemente de su raza, origen étnico, sexo, edad o estado original de hipertensión. Si bien la tensión arterial disminuyó al pasar de una dieta con alto contenido de sodio a una de contenido moderado, la disminución fue aproximadamente dos veces más intensa al pasar de la de contenido moderado a la de contenido bajo. Así que para normalizar la tensión arterial mediante la reducción de la ingesta de sodio, mientras más baja esta, mejor. Los investigadores también recomiendan la dieta DASH de la edad mediana en adelante, por sus efectos beneficiosos cuando aumentan los riesgos de enfermedad cardiovascular. (Bray GA, et al. A further subgroup analysis of the effects of the DASH diet and three dietary sodium levels on blood pressure: results of the DASH-Sodium Trial. Am J Cardiol. 2004;94(2):222-227.)

\section{Efecto del consumo de alcohol y de tabaco sobre la calidad del semen en humanos}

Algunos investigadores han notificado que el consumo de tabaco tiene efectos adversos sobre la concentración, aspecto morfológico y motilidad de los espermatozoides en el semen del ser humano; otros, en cambio, no han detectado efecto alguno. Lo mismo se aplica a los estudios efectuados para determinar si hay o no una relación entre la calidad del semen y el consumo de alcohol. En algunos casos se trata de estudios in vitro o con animales, y en otros, de estudios realizados con muestras pequeñas o en pacientes con problemas de fertilidad. Recientemente un grupo de investigadores en Argentina ha explorado estas relaciones con una muestra de 3976 hombres que fueron divididos en las siguientes categorías: los que no fuman; los que fuman 20 cigarrillos al día o menos; los que fuman más de 20 cigarrillos al día; los que no ingieren be- 